\title{
Paroxysmal nocturnal haemoglobinuria mimicking Crohn's disease
}

Background-The cause of inflammatory bowel disease (IBD) is unknown, and it is often difficult to obtain diagnostic histological specimens. In considering the diagnosis of IBD, other causes of enteritis should be excluded. We present a case that illustrates this.

Case report - A 32 year old man presented with a history of intermittent diarrhoea, vomiting, colicky abdominal pain, and weight loss. There was no history of foreign travel or recent contact with gastroenteritis, and he had no family history or past medical history of note. On examination, he was a pale thin man with central abdominal tenderness; other systems were unremarkable. Abnormal blood tests included a raised white cell count $20.4 \times 10^{6} / 1$ (normal $4-11 \times 10^{6}$ ), C-reactive protein (CRP) $246 \mathrm{mg} / 1$ (normal $1-6 \mathrm{mg} / \mathrm{l}$ ), and ferritin $310 \mu \mathrm{g} / \mathrm{l}$ (normal 12-1200 $\mu \mathrm{g} / \mathrm{l}$ ). Stool cultures were negative for pathogenic organisms. Colonoscopy revealed patchy chronic inflammatory infiltration in the lamina propria and enteroscopy showed active focal duodenititis. In neither case were granuloma seen. An HMPAO labelled leucocyte scan showed grade II uptake in loops of small bowel in the right iliac fossa. On the basis of age, clinical symptoms, and investigations showing inflammation both in small and large bowel, a diagnosis of Crohn's disease was made. Initial treatment included mesalazine and an elemental diet, with resolution of his symptoms and return of CRP to normal.

The patient's illness was subsequently characterised by frequent relapses associated with raised inflammatory indexes and severe abdominal pain requiring opiate analgesia. Trials of prednisolone, ciprofloxacin, azathioprine, and total parenteral nutrition failed to reduce either the frequency or severity of relapses. Further leucocyte scans revealed a variable pattern of disease (fig 1), on one occasion showing multifocal areas of uptake suggestive of peritonitis, without clinical correlation.

His pattern of symptoms gradually changed to include lethargy and episodes of dark urine, associated with abdominal pain. He had a normochromic, normocytic anaemia (haemoglobin $79 \mathrm{~g} / \mathrm{l}$ ) and haematinics were inconclusive. His reticulocyte count was elevated at $4 \%$ (normal 0.8-2\%), with decreased circulating haptoglobins. Urine dipstick was positive for blood, but repeated microscopy revealed no red cells and radiological investigations of the renal tract were normal. Urine haemosiderin was strongly positive, which supported a diagnosis of haemolysis. Coomb's test for autoimmune haemolysis was negative, but immunophenotyping of his peripheral blood cells showed an $80 \%$ loss of glycosyl-phosphatadyl-inositol antigens, which is diagnostic for paroxysmal nocturnal haemoglobinuria $(\mathrm{PNH})$. Supportive measures including blood transfusion, ferrous sulphate supplementation and anticoagulation, have failed to prevent further relapses of severe abdominal pain. Therapeutic options now being considered include bone marrow transplantation.
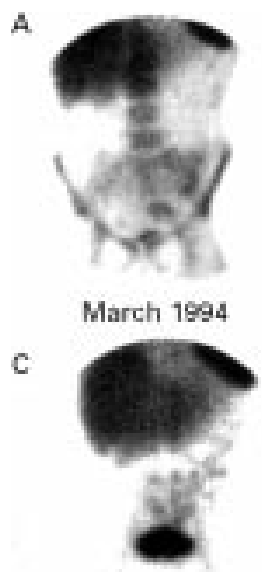

May 1996

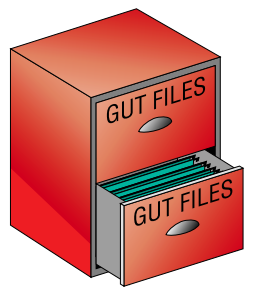

日

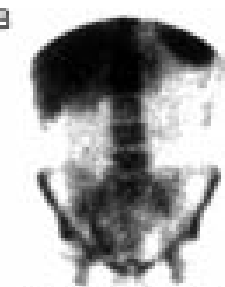

December 1996

D

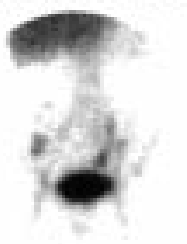

August 1996

Figure 1 (A) Grade II uptake of HMPAO within loops of bowel in the left iliac fossa and pelvis. (B) Grade I inflammation in the pelvis extending towards the right iliac fossa. (C) High irregular tracer uptake in the left side of the abdomen, extending to the right of the midline, suggestive of peritonitis. (D) Irregular inflammatory uptake in the right iliac fossa and left side of the pelvis.

Conclusion-PNH is a clonal disorder of haemopoetic stem cells, resulting in an acquired haemolytic anaemia. The commonest modes of presentation are anaemia alone, abdominal pain, infections, and thrombosis. The abdominal pain is postulated to be caused by microthrombi, haemorrhage into the intestinal wall, or frank intestinal infarction.

This patient's initial presentation was compatible with a diagnosis of Crohn's disease. However, the histological features were non-specific and non-caseating granulomas were never found. Furthermore, his evolving clinical picture, poor response to all modes of treatment, the varying sites of inflammation seen on leucocyte scans, and the association of severe anaemia with enteritis, raised the possibility of alternative pathology. This case highlights the importance of continuing review of the diagnosis of a chronic illness, particularly in the face of changing symptomatology.

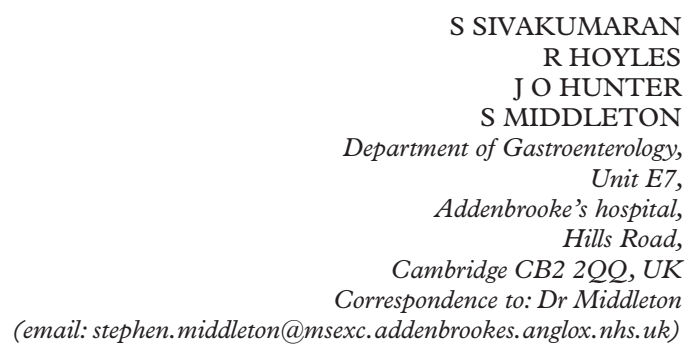

S SIVAKUMARAN

R HOYLES

J O HUNTER

Unit E7,

's hospital,

Cambridge CB2 2OO, UK

(email: stephen.middleton@msexc.addenbrookes.anglox.nhs.uk) 